\title{
The Influence of Social Context and Targeted Communication on e-Government Service Adoption
}

\author{
Michael Räckers, Sara Hofmann, and Jörg Becker \\ European Research Center for Information Systems, University of Münster \\ Leonardo-Campus 3, 48149 Münster, Germany \\ \{michael.raeckers, sara.hofmann, becker\} @ercis.uni-muenster.de
}

\begin{abstract}
The electronic ID card (eID) was introduced in Germany in 2010. Besides the traditional way of authentication, it offers the possibility to activate a function for online authentication. However, more than two thirds of the Germans do not make use of this function. Despite the advantages that the eID offers, the lacking adoption is said to be traced back to lacking information and only few application cases. In our study, we propose online application as a use case for the eID in a university. In a quantitative survey with 1,632 students, we analyse the factors influencing a possible adoption of the eID by students. For this purpose, we extend the basic TAM by the variables perceived risk, social context, experiences, communication and demographics. Our analysis reveals that especially the social context and targeted communication influence the behavioural intention to use and can extremely shape the attitude towards using the service.
\end{abstract}

Keywords: electronic ID card, eID, e-Government, TAM.

\section{Introduction}

After years of debating, the German electronic ID card (eID) was introduced in 2010. [1] Besides being a piece of identification in the 'offline' world, it offers an electronic proof of identity and could also be used for signing documents electronically in the future. Although its launching was accompanied by much public interest, it could not meet the expectations of the government. Until recently, 17 million eID cards have been handed out to German citizens. However, only less than $30 \%$ of the respective citizens have activated the electronic identification function. [2] The number of online applications for the eID is currently limited to 42. [3] Qualified electronic certificates, which are a prerequisite for using the qualified electronic signature functions of the eID, were still in the test phase in December 2012. [4] There are several reasons for the reluctant start of the eID in Germany. On the one hand, the limited number of applications probably causes citizens to ignore possible advantages. On the other hand, security concerns contribute to the rather negative image of the eID. [5] Shortly after the release of the eID card in 2010, the Chaos Computer Club, one of the largest hacker organisations in the world, demonstrated that the reading devices that were distributed together with the card were insecure and could easily be cracked. [6] 
Thus, in order to overcome these barriers and to increase the adoption of the electronic use of the eID, it is crucial to offer services that are consumed by many citizens and that provide an actual benefit for the user. Furthermore, the government should implement a suitable strategy to counteract the reluctant intention to use the new eID card. The arising question is how such a strategy should look like. For answering this question, it is first of all necessary to understand the factors that influence the adoption of the eID.

In order to identify the influencing factors, we propose the application and enrolment process at a German university as a use case for the eID. Universities provide a suitable context for eID studies as many students are likely to possess the new eID card. Furthermore, applying and enrolling for a study is a process all students have to go through. We propose a research model to analyse the variables determining behavioural intention to use the electronic ID card. Our basic hypotheses are based on the Technology Acceptance Model. [7] As proposed by other studies, we extend our research model both by demographic variables, experience, social influence [8] as well as perceived risk [9], [10]. Furthermore, as suggested by previous research [11], we integrate (targeted) communication into our research model. Our resulting research questions are:

RQ1: Which factors influence the behavioural intention to use an eID application by students?

RQ2: Which influence does communication have on the adoption of eID services at universities?

The article is organised as follows. In the next section, we give a short overview of the eID in Germany. Furthermore, we derive our research model based on egovernment respectively IT adoption literature. In Section 3, we describe our research methodology including the design and structure of the questionnaire, the process of data collection as well as of the analysis. This is followed by Section 4, where we present the results of our analysis, and Section 5, in which we discuss the impact of our findings. Section 6 summarises our results and shows both the limits of our study as well as the agenda for future research in this domain.

\section{Related Work}

The eID was introduced in Germany in November 2010 and since then Germany ranks among Belgium, Estonia, Finland, Italy, Portugal and Spain as one of the seven European countries that offer eIDs to their citizens. [12] The German eID provides an electronic proof of identity as well as an electronic signature function. [1] Although the eID is judged as rather secure, the resistance to use them is rather strong. [2]

The aim of our study is to understand both how eID services should be designed as well as how the accompanying communication should take place in order to increase the adoption of these services. Our research model is based on the Technology Acceptance Model (TAM) by [13]. TAM is an often applied model that describes the influence of different variables on IT adoption and that can be adapted easily for different application areas. [14], [15] For our purposes, we use the version of TAM from 1996, which includes the variable perceived usefulness, perceived ease of use, 
behavioural intention and actual system use. [16] As the analysed IT application does not exist yet and as therefore the actual system use (see greyed out box in Fig. 1) cannot be measured, our dependent variable is behavioural intention.

We extend the basic TAM by the factors perceived risk, social influence, communication at the university as well as experiences. Our research model is shown in Fig. 1. Whereas the basic components of TAM are displayed in white rectangles, the added variables are displayed in light grey ones. The variables on the dark grey background are tested for their dependence on demographic factors (age, gender, computer skills, study programme). The assumed relations are shown by arrows.

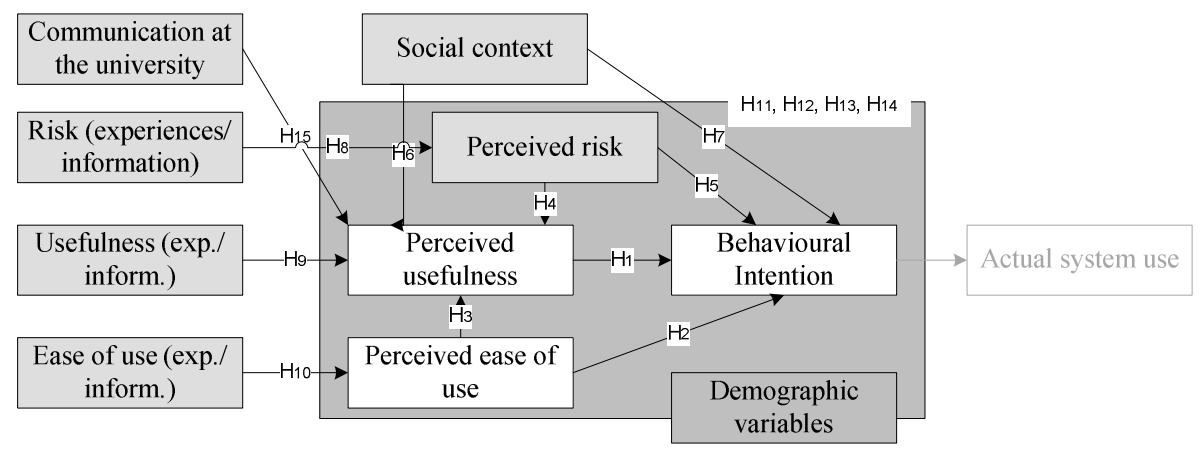

Fig. 1. Research model for the adoption of eID services at the university

Basic model: The basic model of TAM has been used in a variety of studies dealing with the adoption of IT since then. [16] TAM explains the actual use of a system as being influenced by the behavioural intention to use the system, which in turn is influenced by perceived usefulness as well as perceived ease of use. Furthermore, perceived ease of use also impacts perceived usefulness. Despite its popularity, TAM has been subject to a lot of criticism due to lacking objective antecedents as well as "black box" concepts. [17] However, as it is one of the most tested and most successful theories in IT adoption and also in e-government adoption (cf. e.g. [10], [18]), we base our basic hypotheses on the assumptions of TAM:

\section{$H_{1}$ : Perceived usefulness will positively influence behavioural intentions. \\ $\mathrm{H}_{2}$ : Perceived ease of use will positively influence behavioural intentions. \\ $H_{3}$ : Perceived ease of use will positively influence perceived usefulness.}

Perceived risk: In the research area of IT adoption and especially in e-government adoption, the notion of (perceived) risk plays an important role as many sensitive data is processed [11] as well as due to the impersonality of transactions on the internet [19]. In the context of e-government, perceived risk is understood as "the citizen's subjective expectation of suffering a loss in pursuit of a desired outcome". [20] Previous research on e-government acceptance suggests that perceived risk has a negative impact on perceived usefulness and behavioural intention. [9], [21], [22] In our survey, we especially focus on security concerns in terms of privacy and data security. 


\section{$H_{4}:$ Perceived risk will negatively influence perceived usefulness.}

$H_{5}$ : Perceived risk will negatively influence behavioural intentions.

Social context: A further concept that has been analysed in IT adoption studies is social context. [23] identified a relation between social influence and behavioural intention. This hypothesis has been studied in different e-government specific studies, too. (cf. e.g. [24]) Further research suggests a correlation between social context and the attitudes of a system user [25], which is especially the case for social context and perceived usefulness. [26]

$H_{6}:$ The social context will positively influence perceived usefulness.

$H_{7}$ : The social context will positively influence behavioural intentions.

Experiences: Based on the Unified Theory of Acceptance and Use of Technology (UTAUT), an extension of TAM [8], we assume a correlation between the previous assessment of the eID based on experiences and information and perceived risk, perceived usefulness and perceived ease of use.

$H_{8}$ : The previous assessment of risk based on experiences and information on the eID will positively influence perceived risk.

$H_{9}$ : The previous assessment of perceived usefulness based on experiences and information on the eID will positively influence perceived usefulness.

$H_{10}$ : The previous assessment of perceived ease of use based on experiences and information on the eID will positively influence perceived ease of use.

Demographics: Demographic factors have been identified as influencing factors in previous studies of IT acceptance. [27], [28] In our case, we analyse the impact of age, gender, field of study and computer skills on perceived risk, perceived usefulness, perceived ease of use and behavioural intention.

$H_{11}$ : Age will influence perceived risk, perceived usefulness, perceived ease of use and behavioural intention.

$H_{12}$ : Gender will influence perceived risk, perceived usefulness, perceived ease of use and behavioural intention.

$H_{13}$ : The field of study will influence perceived risk, perceived usefulness, perceived ease of use and behavioural intention.

$H_{14}$ : Computer skills will influence perceived risk, perceived usefulness, perceived ease of use and behavioural intention.

Communication: A further question is how communication influences the adoption of IT. Therefore, we analyse whether targeted communication content and channels can influence the attitude towards the eID. Communication can play a vital role in spreading information. [29] Furthermore, previous research suggests that lacking communication is one reason for reluctant e-government adoption in Germany. [11]

$H_{15}$ : Targeted communication at university on the eID will influence perceived usefulness. 


\section{$3 \quad$ Methodology}

In most studies, technology acceptance has been researched using surveys. [8], [9] As we propose a quantitative model to understand the influencing factors of the adoption of the eID card, our instrument was a standardised online questionnaire.

We tested our hypotheses in the context of an application case for the eID card at a German university. In collaboration with the university's IT service provider as well as based on an online research, we identified several possible services in which an eID card could be used. We presented these possible applications in our questionnaire asking the participants for their assessment. Our items were developed from previous studies of e-government respectively IT adoption.

Our survey was structured as follows: The first part contained the introduction and introductory questions in which we gave an overview of the topic and asked general information about the participant. This was followed by questions on the assessment of the eID concerning perceived ease of use, perceived risk and perceived usefulness as well as questions concerning the frequency of eID usage and the level of satisfaction. Afterwards, we presented possible applications. We identified the services Single sign-on (SSO) and signing forms electronically as well as the application and enrolment process. Participants were asked for their assessment of the variables of our research model. The item scale for our constructs was a four endpoint scale (ranging from strongly agree to strongly disagree). Afterwards, we recorded the information need and the influence of communication by querying which factors the participants would like to be informed about through which channels (assuming that the eID service was implemented at the university). In the final part, we collected demographic data and allowed for comments. Completing the survey took about 15 minutes. As an incentive to take part, we gave away three Amazon gift cards.

We pre-tested the survey in several rounds. First of all, an expert from the IT service provider checked it for logical mistakes. We conducted the first round of pretests while we were present. The second round of pre-tests was sent via e-mail. As this only led to few changes, we launched the survey afterwards. We sent an invitation link to the online survey to all employees and students of the university via e-mail, which are about 45,000 persons in total. Filter questions ensured that the different target groups would only answer questions relevant to them.

We analysed our data with the aid of Excel for descriptive statistics as well as with SPSS for correlation analysis and linear regression. Besides calculating the Spearman correlation, we ran a regression analysis for the influencing factors of behavioural intention and perceived usefulness.

\section{$4 \quad$ Data and Results}

The questionnaire was implemented in an online survey tool and was open from June $26^{\text {th }}$ to July $10^{\text {th }}$. Overall, 1,632 students participated and completed the questionnaire. $37 \%$ of the participants stated that they have the new ID card, 35\% of these additionally have activated the electronic functionalities of the eID and $29 \%$ have an 
appropriate card reader to use these functionalities. This is in line with the number of new eIDs and released electronic functionalities in Germany in general. [30]

One important aspect for the evaluation of the results was the level of information the participants have regarding the eID. In all aspects we asked for, less than $25 \%$ of the participants answered that they are very good or good informed (cf. Table 1).

Table 1. Level of information about the eID card (1=very good to $4=$ not informed)

\begin{tabular}{|l|c|c|c|c|}
\hline Level of information & 1 & 2 & 3 & 4 \\
\hline eID functionality & $3.01 \%$ & $15.82 \%$ & $42.24 \%$ & $38.93 \%$ \\
\hline eSign functionality & $4.50 \%$ & $20.12 \%$ & $36.10 \%$ & $39.28 \%$ \\
\hline Security aspects & $2.97 \%$ & $14.58 \%$ & $34.48 \%$ & $47.97 \%$ \\
\hline Fields of application & $4.36 \%$ & $14.14 \%$ & $34.31 \%$ & $47.18 \%$ \\
\hline General level of information & $2.45 \%$ & $13.72 \%$ & $50.07 \%$ & $33.77 \%$ \\
\hline
\end{tabular}

For assessing the influence of the social context on the behavioural intentions one main aspect was the importance of different sources for information about the eID card in general. We did not divide the sources for information further in subsets like source for information for applications or for security aspects. Regarding the source of information the participants build their own opinion on, the results show that they count on official information and information of newspapers as well as information from their direct social environment like family, friends or colleagues (cf. Table 2).

Table 2. Source of Information, the participants build their own opinion on (1=very important to $4=$ not important)

\begin{tabular}{|l|c|c|c|c|}
\hline & 1 & 2 & 3 & 4 \\
\hline Official sources & $46.00 \%$ & $38.22 \%$ & $11.67 \%$ & $4.11 \%$ \\
\hline Newspapers & $22.21 \%$ & $49.89 \%$ & $21.99 \%$ & $5.90 \%$ \\
\hline User suggestions & $10.98 \%$ & $25.30 \%$ & $36.37 \%$ & $27.35 \%$ \\
\hline Suggestions of family and friends & $21.12 \%$ & $46.33 \%$ & $25.38 \%$ & $7.16 \%$ \\
\hline
\end{tabular}

In the following we will exemplary concentrate on the last case as the results for all three cases are by trend the same. Overall, $58 \%$ of the students state that they would use the eID card for an online application at the university (categories 1 and 2 of 4 on a scale from "most likely" to "no") and 19\% would not use eID card for a completely electronic application process (category 4). Furthermore, 57\% of the students think that their family or friends would use the eID card for the application process and $12 \%$ think that their family or friends would not use it. Condensing the further questions regarding the scenario of complete online application and calculating the correlation based on Spearman we determine an influence for every of our hypotheses $\mathrm{H}_{1}$ to $\mathrm{H}_{10}$ (cf. Fig. 2 and Table 3). 


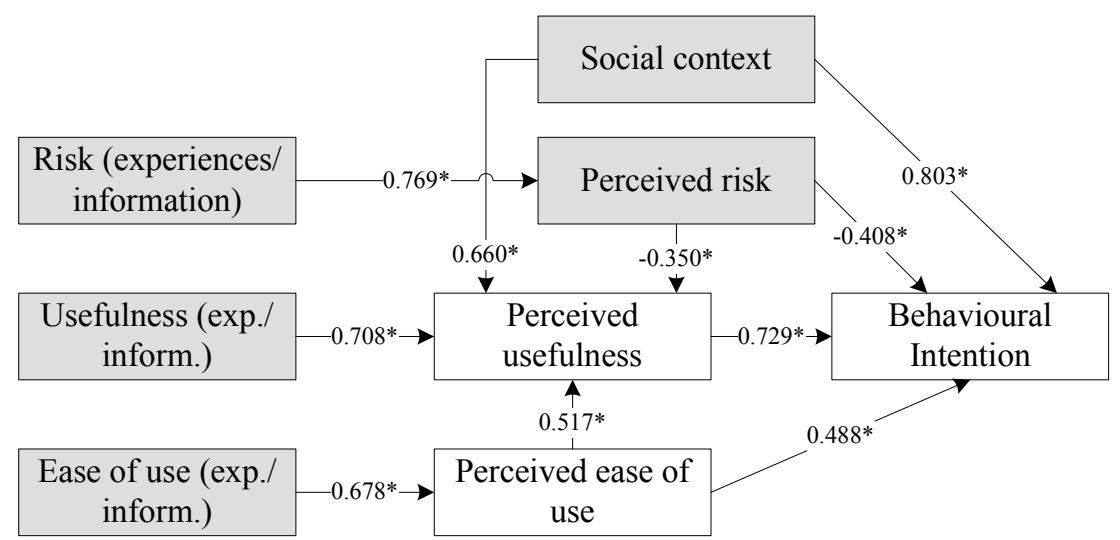

Fig. 2. Spearman Correlation of factors on behavioural intention of students to use the eID card for online application for a university place $(0.01 \%$ level $)$

Table 3. Spearman Correlation for scenario of complete online application for a university place $\left(0.01 \%\right.$ level) for hypotheses $\mathrm{H}_{1}$ to $\mathrm{H}_{10}$

\begin{tabular}{|l|r|r|r|r|r|r|r|r|r|r|}
\hline Hypothesis & $\mathrm{H}_{1}$ & $\mathrm{H}_{2}$ & $\mathrm{H}_{3}$ & $\mathrm{H}_{4}$ & $\mathrm{H}_{5}$ & $\mathrm{H}_{6}$ & $\mathrm{H}_{7}$ & $\mathrm{H}_{8}$ & $\mathrm{H}_{9}$ & $\mathrm{H}_{10}$ \\
\hline Spearman & 0.729 & 0.488 & 0.517 & -0.350 & -0.408 & 0.660 & 0.803 & 0.743 & 0.708 & 0.678 \\
\hline
\end{tabular}

Additionally, we performed a regression analysis for perceived usefulness and behavioural intention to use the eID card. Perceived usefulness has an R2 of 0.722 . The social context has a high influence on the perceived usefulness; the perceived risk has a small influence on the perceived usefulness. Additionally, the usefulness in general also has a high influence. BBehavioural intention also shows an R2 of 0.722 . Here, too, perceived risk has a rather small influence while the influence of perceived usefulness and of social context have a high influence. Additionally, perceived ease of use does not have a noticeable influence on behavioural intention to use the eID card for the online application for a university place.

From the set of demographic aspects, only the individual computer skills have a significant influence on behavioural intention to use. However, even in this category, behavioural intention to use the eID for online application only slightly depends on the level of computer skills as it ranked smoothly between $61 \%$ (very good skills) and $50 \%$ (low skills)

We asked for indicators of perceived usefulness in every usage scenario. Based on the hypothetical process of a complete-online application, the students said that it would be a useful, easy and fast alternative to the classical, (partly) paper-based way. However, they do not see any improvements in safety issues and are indifferent regarding financial issues (cf. Table 4).

Finally, we asked for communication aspects to prove the influence of communication on the behavioural intention to use the German eID. Overall, about $60 \%$ of the students indicated that they (most) likely think that their attitude regarding the eID could be influenced through communication of the university (cf. Table 5). 
Table 4. Perceived usefulness of the eID card for online application at university $(1=$ strongly agree to $4=$ disagree)

\begin{tabular}{|l|l|l|l|l|}
\hline & 1 & 2 & 3 & 4 \\
\hline Useful alternative & $35.43 \%$ & $34.70 \%$ & $16.00 \%$ & $13.87 \%$ \\
\hline Fast alternative & $40.28 \%$ & $37.04 \%$ & $13.51 \%$ & $9.17 \%$ \\
\hline Easy alternative & $24.95 \%$ & $40.53 \%$ & $21.15 \%$ & $13.37 \%$ \\
\hline Safer alternative & $8.11 \%$ & $18.68 \%$ & $36.42 \%$ & $36.79 \%$ \\
\hline Cheap alternative & $18.84 \%$ & $30.81 \%$ & $24.82 \%$ & $25.53 \%$ \\
\hline
\end{tabular}

Table 5. Possible influence by university's communication about eID ( $1=$ most likely to $4=$ no)

\begin{tabular}{|l|c|c|c|c|}
\hline & 1 & 2 & 3 & 4 \\
\hline $\begin{array}{l}\text { Possible influence by communication } \\
\text { about eID through university }\end{array}$ & $10.44 \%$ & $49.72 \%$ & $29.33 \%$ & $10.51 \%$ \\
\hline
\end{tabular}

Further results show that students wish to be informed by university about security aspects of the eID (80\%), university's online services for the eID card (72\%) as well as about the general functionality (72\%). Only $10 \%$ do not want to get information about the eID card by their university. Regarding the preferred channel, students want to be informed by mail $(74 \%)$ as well as the universities website $(74 \%)$ or the website of the universities IT provider $(66 \%)$. They do not want to get information via the social websites of the university (e.g., the Facebook page of the university).

\section{Discussion}

It is striking, that the results show a low level of awareness about functionality and fields of application of the new eID card but a significantly high interest in this new technology resp. services. To add, this interest in this rather new technology even only slightly depends on individual technical skills.

However, the low level of awareness firstly has to be ascribed to the governmental level as governments should have a high interest in informing the people about the functionality and services of the eID. If students increase using their eID card for university services and provide all their information and applications digitally, process costs within the universities will be reducible in the future. This would not only be true for universities but also for other organizations with high rates of document processing like governments, insurance companies, or banks. They all provide various services which would benefit from complete digital processing. It would help to optimize business processes to provide faster and thus cheaper services to their customers.

Furthermore, our results show that the participants prefer several channels for information about the eID card and services around. They not only seek for information from official sources or newspapers, but rely heavily on the opinion of the social environment about the eID card and its related services. Within our correlation analysis the influence of the social context on the behavioural intention to use the eID card for the online application is very strong, which is conform to 
previous research. This is an important finding as this should have an influence on the information and communication strategies of the stakeholders of the eID either being the governments or being further providers for services around the eID like a university.

It is striking that only one third of the citizens which have a new eID card activate the electronic functionalities. This reduces the actual amount of potential users for services relying on the eID functionalities to only about roughly $10 \%$. This, furthermore, raises the question about the reasons for this low adoption rate. Recent research shows that various aspects have an influence on this acceptance rate. Besides technical, environmental, service and user characteristics, trust and communication have to be mentioned here. [31]

Our results presented in Section 4 show, that the ease of use (technical and service characteristics), the social context and skills (environmental and user characteristics), security issues (trust) and communication have a significant influence of the behavioural intention to use the eID card at university. To underline this, the participants furthermore explicitly mentioned security concerns as main reasons for not using the eID card, the feeling of less information and the fear of increase in complexity. However, based on our results, many of the participants see the eID card as a useful, fast and easy alternative to the actual way of applying for a university position. This shows the importance of targeted communication efforts to explain the potentials users the advantages of the new technologies.

Of course, we cannot transfer this impression of ease of use directly to other services ready for eID cards. However, in our example case it underlines the importance of the further influencing factors, i.e. trust and the already mentioned targeted communication. From our perspective these two factors interrelate to some extent. On the one hand, for sure, the eID itself as well as the services build upon the eID card have to be secure in terms of privacy of data and protection of data loss, which goes along with further results [9], [21], [22]. On the other hand, the central government needs to declare that the underlying techniques are safe. One interpretation based in this could be that the central government is either not trustable from a citizens point of view and citizens do not believe in the communication of the governments or the central government is not able to communicate the security of their techniques and services in the right way. Following on this service providers like a university also encounter problems in communicating the safety of the technology. Recent research thoroughly allows for the conclusion that there are deficits in the communication efforts of governments. [11] Therefore, improving the way of communication would help to increase the acceptance rate of eID-supported services.

Additionally, our results show that it is not only important that the central government as provider of the eID card informs and communicates the possibilities, services or security aspects. It is also up to the university as (potential) provider of concrete services itself to inform about these aspects and provide information about the single service as well as the overall techniques behind.

To sum up on this, a holistic concept is necessary in which the information strategies of the central government and the university as representative for the single service providers are aligned. Our results show that the citizens, i.e. the students, feel uninformed about the possibilities of the eID card. Additionally, this concept has to 
reflect the importance of the social environment of every single (potential) user. For example this leads to a somehow grassroots approach, were users are stimulated to foster their friends and colleagues to also use the eID card for services available. The university resp. the service providers here have the advantage that they are to some extent closer to the customers. It is easier for them to perform targeted communication, dedicated to the special needs of the (potential) users. Close interrelation between these stakeholders is obligatory. From our perspective, this could be one way to breach the chicken and egg problem we are facing. On the one hand there are less (potential) users because of few citizens activating the eID functionality. So actually it is not that promising for service providers like the university to adapt their authentication services to the eID card. On the other hand citizens are hesitating to activate this functionality because of only few services actually available, neither at university nor at public administrations.

\section{Conclusion}

Our analysis pretty well shows the actual dilemma the central government and service providers which offer the new eID card and services related are facing. On the one side the problem of few services available and on the other side potential users struggling to activate and use the services because of less possibilities to use. One main reason which could be synthesized is the feeling of lacking information the students participated in our survey expressed. So our main conclusions and answer to our research questions are that a better, targeted communication and the inclusion of the social context of the (potential) users could be key factors for improvement.

As such our study contributes to the actual body of knowledge. We were able to confirm related work in technology acceptance research in confirming and extending the existing theories and models, especially in our case research related to the TAM. We especially focussed on the aspects of communication and the social context of the users. Additionally, as implication for practice, we synthesized from data that central government together with their aligned service providers have to extend their communication concepts, targeted to single user groups, to enhance their actual efforts and achieve better results in terms of eID adoption. We especially were able to prove the strong influence of the social context on the attitude towards eID services.

Our study has a number of limitations which should be followed by further research. We focussed on the field of services combined with an eID card in the university context. Furthermore, we limited or work to exemplary services within a university environment. On the one hand this limits the generalization of our findings; on the other hand this was necessary to handle the complexity of the field to a level which was understandable for the attendees of the survey.

Further research should flow in two main directions. Firstly, the results achieved in the university context have to be extended to further services which are relevant for an eID card application. This has to be done in the university area as well as for services of public administrations and private organizations. The purpose would be to further approve the findings and make them generalizable. Secondly, the aspects of 
communication and the inclusion of the social context into the communication strategies and concepts have to be conducted and applied. This application subsequently has to be evaluated from a scientific perspective to ensure an integrated prove of the findings of our work.

Acknowledgements. We would like to thank Adrian Beheschti, Lisa Eckey, Heinrich Hüppe, Magdalena Lang, Philipp Simon, Fritz Ulrich, Patrick Vogel and Till Weber for their dedicated effort in conducting the study.

\section{References}

1. Hornung, G., Roßnagel, A.: An ID card for the Internet - The new German ID card with 'electronic proof of identity. Computer Law \& Security Review 26(2), 151-157 (2010)

2. Keitzel, O., Zimmer, W., de Vries, E., Kubicek, H., Wind, M.: Mittelstandsoffensive Neuer Personalausweis. Expertise und Handlungsempfehlungen für die Etablierung zentraler eID-Infrastrukturen für den Mittelstand, Berlin (2012)

3. Federal Ministry of the Interior, Der neue Personalausweis (2012), http: / /www.personalausweisportal.de/DE/Home/home_node.html (accessed: December 06, 2012)

4. Kirsch, C.: Digitale Signatur mit dem ePerso zum Sonderpreis (2012), http: / /www. heise.de/newsticker/meldung/Digitale-Signaturmit-dem-ePerso-zum-Sonderpreis-1751434.html (accessed: December 06, 2012)

5. Grote, J.H., Keizer, D., Kenzler, D., Kenzler, P., Meinel, C., Schnjakin, M., Zoth, L.: Vom Client zur App: Ideenkatalog zur Gestaltung der Software (2010)

6. Kirk, R.: Hacking of new German ID card. Managing Information 17(7), 26 (2010)

7. Davis, F.D., Bagozzi, R.P., Warshaw, P.R.: User Acceptance of Computer Technology: A Comparison of Two Theoretical Models. Management Science 35(8), 982-1003 (1989)

8. Venkatesh, V., Morris, M., Davis, G.: User acceptance of information technology: Toward a unified view. MIS Quarterly 27(3), 425-478 (2003)

9. Bélanger, F., Carter, L.: Trust and risk in e-government adoption. The Journal of Strategic Information Systems 17(2), 165-176 (2008)

10. Carter, L., Bélanger, F.: The utilization of e-government services: citizen trust, innovation and acceptance factors. Information Systems Journal 15(1), 5-25 (2005)

11. Hofmann, S., Heierhoff, L.: Adoption of Municipal e-Government Services - A Communication Problem? In: Proceedings of the 18th Americas Conference on Information Systems, AMCIS 2012, p. Paper 7 (2012)

12. European Union, Electronic identification, signatures and trust services: Questions and Answers (2012), http://europa.eu/rapid/press-release_MEMO-12403_en.htm (accessed: December 07, 2012)

13. Davis, F.D., Bagozzi, R.P., Warshaw, P.R.: User Acceptance of Computer Technology: A Comparison of Two Theoretical Models. Management Science 35(8), 982-1003 (1989)

14. Pikkarainen, T., Pikkarainen, K., Karjaluoto, H., Pahnila, S.: Consumer acceptance of online banking: an extension of the technology acceptance model. Internet Research 14(3), 224-235 (2004) 
15. Zhou, Y.: Voluntary adopters versus forced adopters: integrating the diffusion of innovation theory and the technology acceptance model to study intraorganizational adoption. New Media \& Society 10(3), 475-496 (2008)

16. Venkatesh, V., Davis, F.D.: A Model of the Antecedents of Perceived Ease of Use: Development and Test. Decision Sciences 27(3), 451-481 (1996)

17. Benbasat, I., Barki, H.: Quo Vadis, TAM? Journal of the Association for Information Systems 8(4), 211-218 (2007)

18. Sipior, J.C., Ward, B.T., Connolly, R.: The digital divide and t-government in the United States: using the technology acceptance model to understand usage. European Journal of Information Systems 20(3), 308-328 (2011)

19. Pavlou, P., Tan, Y.-H., Gefen, D.: Institutional trust and familiarity in online interorganizational relationships. In: Proceedings of the 11th European Conference on Information Systems (2003)

20. Warkentin, M., Gefen, D., Pavlou, P., Rose, G.: Encouraging citizen adoption of egovernment by building trust. Electronic Markets 12(3), 157-162 (2002)

21. Horst, M., Kuttschreuter, M., Gutteling, J.M.: Perceived usefulness, personal experiences, risk perception and trust as determinants of adoption of e-government services in The Netherlands. Computers in Human Behavior 23(4), 1838-1852 (2007)

22. Featherman, M.S., Pavlou, P.: Predicting e-services adoption: a perceived risk facets perspective. International Journal of Human-Computer Studies 59(4), 451-474 (2003)

23. Venkatesh, V., Morris, M.G., Davis, G., Davis, F.D.: User acceptance of information technology: toward a unified view. MIS Quarterly 27(3), 425-478 (2003)

24. Gupta, B., Dasgupta, S., Gupta, A.: Adoption of ICT in a government organization in a developing country: An empirical study. The Journal of Strategic Information Systems 17(2), 140-154 (2008)

25. Guo, Z., Stevens, K.J.: Factors influencing perceived usefulness of wikis for group collaborative learning by first year students. Australasian Journal of Educational Technology 27(2), 221-242 (2011)

26. Shen, D., Laffey, J., Lin, Y., Huang, X.: Social Influence for Perceived Usefulness and Ease-of-Use of Course Delivery Systems. Journal of Interactive Online Learning 5(3), 270-282 (2006)

27. Agarwal, R., Prasad, J.: A Conceptual and Operational Definition of Personal Innovativeness in the Domain of Information Technology. Information Systems Research 9(2), 204-215 (1998)

28. Gefen, D., Straub, D.W.: Gender Differences in the Perception and Use of E-Mail: An Extension to the Technology Acceptance Model. Management Information Systems Quarterly 21(4), 389-400 (1997)

29. Rogers, E.M.: Diffusion of Innovations. The Free Press, New York (1995)

30. Borchers, D.: Zwei Jahre später: der neue Personalausweis (2012), http: / / www . heise. de/newsticker/meldung/Zwei-Jahre-spaeterder-neue-Personalausweis-1736387.html (accessed: December 07, 2012)

31. Hofmann, S., Räckers, M., Becker, J.: Identifying Factors for e-Government Acceptance a Literature Review. In: Proceedings of the International Conference on Information Systems, ICIS 2012 (2012) 